\title{
Pigmented paravenous retinochoroidal atrophy: a case report
}

\author{
Yinchen Shen, Xun Xu and Hui Cao
}

\begin{abstract}
Background: Pigmented paravenous retinochoroidal atrophy (PPRCA) is an unusual retinal degeneration, and its performance on optical coherence tomography angiography (OCTA) is unclear. We report a Chinese female case of PPRCA and her OCTA features.

Case presentation: A 66-year-old female patient was referred to the author's center for gradual progressive loss of vision in both eyes and photophobia of 2 years duration. She reported having no family history of inherited ocular diseases. The funduscopic examination revealed bone-spicule pigmentation and retinochoroidal atrophy along the retinal veins. This patient was diagnosed with PPRCA which is a rare disease, uncommon in females, more commonly affecting the paravascular fundus. Noninvasive imaging techniques features of this patient was described, including ultra-wide field fundus autofluorescence, spectral domain optical coherence tomography (SD-OCT), OCTA (SSADA), etc. The en face OCTA images demonstrated areas of flow void beneath the retinal pigment epithelium-Bruch membrane layer suggestive of choriocapillaris hypoperfusion that corresponded with indocyanine green angiography (ICGA). Further studies should be conducted to clarify the relationship between choriocapillaris hypoperfusion and the development of PPRCA.

Conclusions: The OCTA features in patients with PPRCA has not been described previously in the literature. This case might provide preliminary information regarding the pathophysiology of PPRCA and improve our understanding of the nature of this disease.

Keywords: Pigmented paravenous retinochoroidal atrophy, Fundus autofluorescence, Spectral domain optical coherence tomography, Optical coherence tomography angiography, Electroretinogram
\end{abstract}

\section{Background}

Pigmented paravenous retinochoroidal atrophy (PPRCA) is an unusual retinal degeneration characterized by perivenous aggregations of pigment clumps associated with peripapillary and radial zones of retinochoroidal atrophy that are distributed along the retinal veins [1]. Patients are often asymptomatic and the diagnosis is based on a characteristic fundus appearance [2]. The etiology remains unknown or idiopathic, although many report of familial cases make hereditary nature of this condition quite apparent [3]. We here report a Chinese female case of PPRCA.

\footnotetext{
* Correspondence: 13611878111@126.com

Department of Ophthalmology, Shanghai General Hospital, Shanghai Jiao Tong University School of Medicine (originally named "Shanghai First People's Hospital"), No. 100 Haining Road, Shanghai 200080, People's Republic of China
}

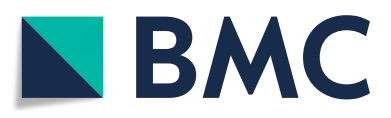

(อ) The Author(s). 2018 Open Access This article is distributed under the terms of the Creative Commons Attribution 4.0 International License (http://creativecommons.org/licenses/by/4.0/), which permits unrestricted use, distribution, and reproduction in any medium, provided you give appropriate credit to the original author(s) and the source, provide a link to the Creative Commons license, and indicate if changes were made. The Creative Commons Public Domain Dedication waiver (http://creativecommons.org/publicdomain/zero/1.0/) applies to the data made available in this article, unless otherwise stated.

\section{Case presentation}

A 66-year-old female patient was referred to the authors' center for gradual progressive loss of vision in both eyes and photophobia of 2 years duration. She reported having no family history of inherited ocular diseases, no history of trauma, no history of inflammation or infectious diseases. Her best corrected visual acuity (BCVA) was 65 ETDRS letters (Snellen equivalent 20/50) in the right eye and 61 ETDRS letters (Snellen equivalent 20/62.5) in the left eye. The anterior segment was unremarkable in both eyes. No relative afferent pupillary defect was found. The intraocular pressure was normal. Funduscopic examination after pupillary dilation found bone-spicule pigmentation and retinochoroidal atrophy along the retinal veins, bilaterally, without macular involvement or signs of inflammation.

Ultra-wide field fundus autofluorescence imaging revealed demarcated hypoautofluorescent areas corresponding to the atrophic patches in the peripheral paravenous distribution, surrounded by a relatively hyperautofluorescent band (Fig. 1). 


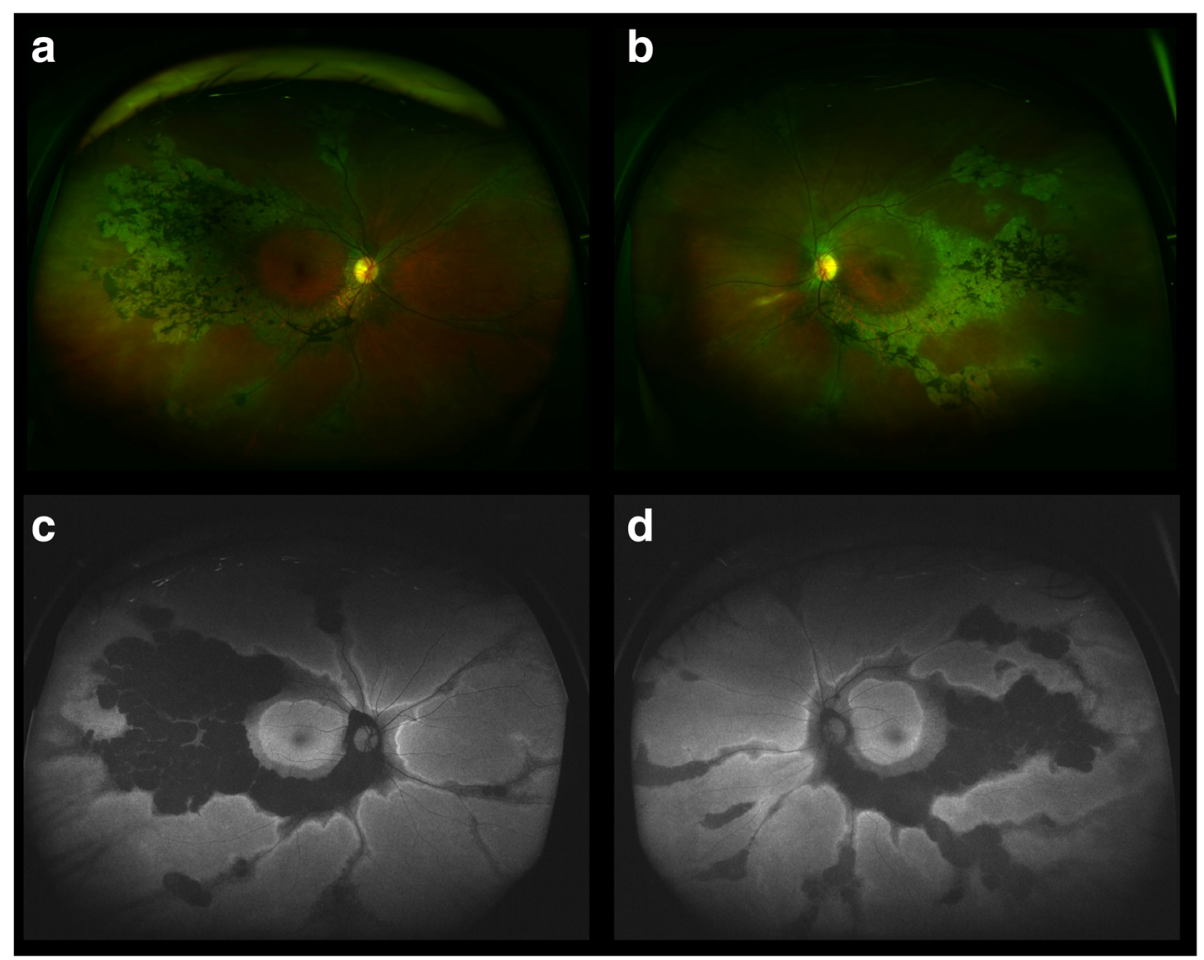

Fig. 1 Ultra-wide field fundu photography showed bone-spicule pigmentation and retinochoroidal atrophy in the peripheral paravenous distribution, bilaterally, without macular involvement (right eye $\mathbf{a}$; left eye $\mathbf{b}$ ). Fundus autofluorescence imaging revealed sharply demarcated hypoautofluorescent areas corresponding to the atrophic patches along the vein, surrounded by a relatively hyperautofluorescent band (right eye $\mathbf{c}$; left eye $\mathbf{d}$ )

Spectral domain optical coherence tomography (SD-OCT) imaging found an obvious thinning of the entire outer retina, but the microstructure of the macular remained intact (Fig. 2). Fluorescein angiography (FA) and indocyanine green angiography (ICGA) showed a window defect with visualization of medium-to-large-caliber choriocapillary vessels and hypofluorescence, respectively (Fig. 3), corresponding to the atrophic area along the veins and the optic disc. FA also show staining along borders of atrophic areas.
In order to better reveal the subtle abnormalities that may not be detectable on routine FA and ICGA, $6 * 6 \mathrm{~mm}$ optical coherence tomography angiography (OCTA, SSADA) images were acquired to capture more information. Within the choroid capillary layer, the en face OCTA images demonstrated areas of flow void beneath the retinal pigment epithelium-Bruch membrane layer suggestive of choriocapillaris hypoperfusion that corresponded with ICGA, and there also appear to be more large-caliber
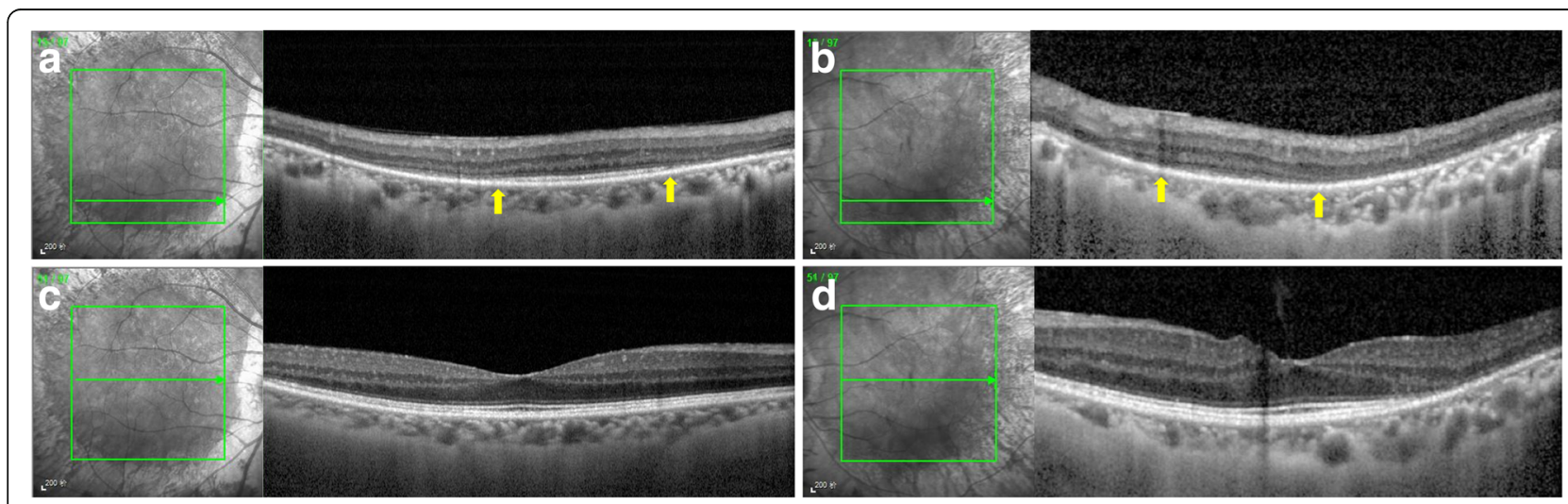

Fig. 2 Spectral domain optical coherence tomography (SD-OCT) imaging found an obvious thinning of the entire outer retina (yellow arrow) including outer nuclear layer, external limiting membrane, myoid zone, ellipsoid zone, and interdigitation zone in the paravenous areas (right eye $\mathbf{a}$; left eye $\mathbf{b}$ ), but the microstructure of the macular remained intact (right eye $\mathbf{c}$; left eye $\mathbf{d}$ ) 


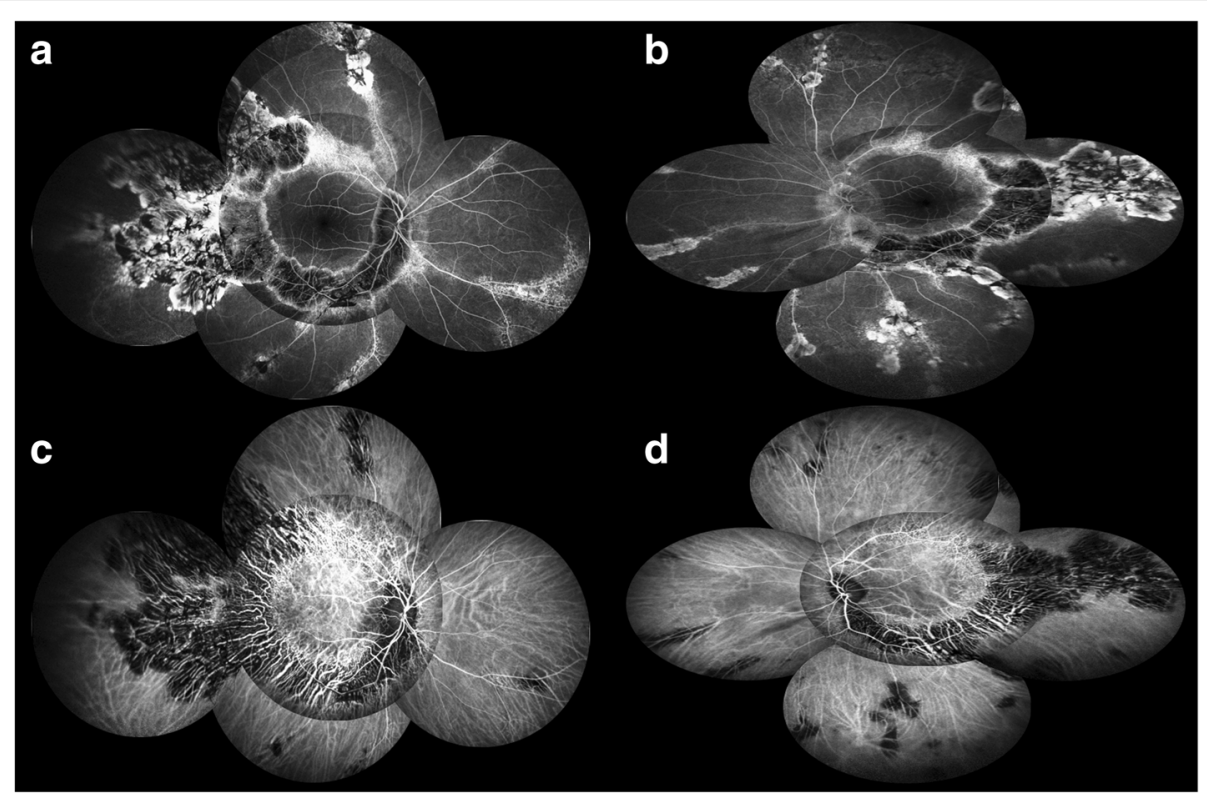

Fig. 3 Fluorescein angiography (FA) and indocyanine green angiography (ICGA) showed a window defect (a, b) with visualization of medium-to-largecaliber choriocapillary vessels and hypofluorescence $(\mathbf{c}, \mathbf{d})$, respectively, corresponding to the atrophic area along the veins and the optic disc. FA also show staining along borders of atrophic areas
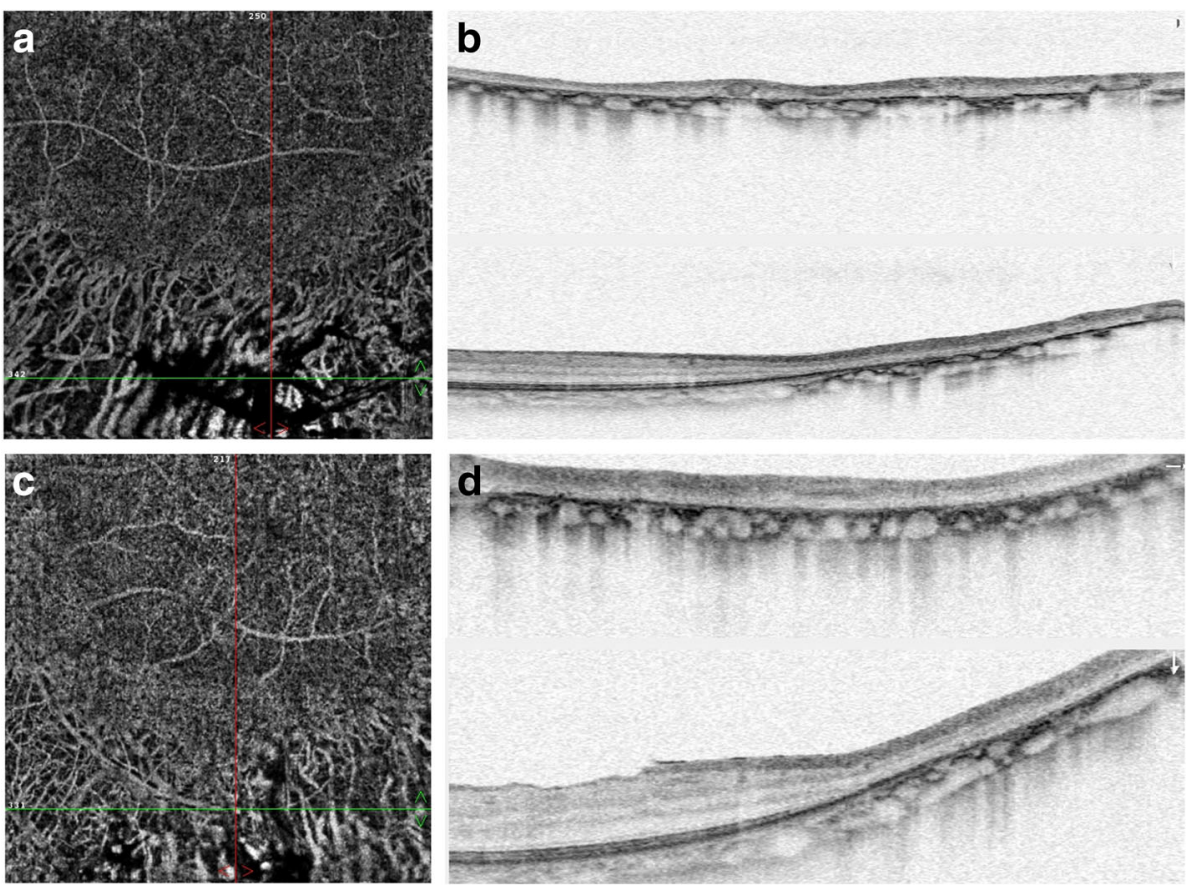

Fig. 4 Within the choroid capillary layer, the en face OCTA images demonstrated areas of flow void beneath the retinal pigment epithelium-Bruch membrane layer suggestive of choriocapillaris hypoperfusion that corresponded with ICGA, and there also appear to be more large-caliber vessels in areas of choriocapillary loss (a and $\mathbf{c}$ were the en face OCTA images within the choroid capillary layer for the right eye and the left eye, respectively; $\mathbf{b}$ and $\mathbf{d}$ were the horizontal and vertical OCT scans for the right eye and the left eye, respectively) 
vessels in areas of choriocapillary loss. (Fig. 4). Electroretinogram (ERG) of both eyes showed mildly subnormal responses. A diagnosis of PPRCA was arrived at. The nature of the disease its prognostic was explained to the patient.

\section{Discussion}

PPRCA is a rare ocular disease with unknown etiology and slow progression. For this patient, the cause of the disease still remains unclear. Although the diagnosis of PPRCA is based on typical fundus appearance, the findings of fundus autofluorescence, FA, ICGA, and ERG can help us to confirm the diagnosis.

In this case, OCTA is applied to analyze the vascular structures of the retina and the choroid to reveal subtle abnormalities. OCTA is a novel imaging technique that provides noninvasive angiographic maps of the retinal and choroidal vasculature. The technique of OCTA is based on split-spectrum amplitude-decorrelation angiography that is able to detect endoluminal flow and reconstruct the retinochoroidal microvascular network [4]. OCTA images of our patient demonstrated areas of flow void suggestive of choriocapillaris hypoperfusion, which may lead to insufficient metabolic supplementation for the outer retinal structures [5], but hypoperfusion of the choriocapillars could also result from RPE/ outer retinal loss. Therefore, it is unclear whether choroidal blood flow diminution is a etiology or a coexisting finding in this case. Further studies should be conducted to clarify the relationship between choriocapillaris hypoperfusion and the development of PPRCA.To our knowledge, the OCTA features in patients with PPRCA has not been described previously in the literature, and this case report might provide preliminary information regarding the pathophysiology of PPRCA and improve our understanding of the nature of this disease.

\section{Conclusions}

In summary, we reported a Chinese female case of PPRCA and the multimodal imaging of this patient. To the best of our knowledge, this is the first case to report the OCTA features of a PPRCA patient.

\footnotetext{
Abbreviations

BCVA: Best corrected visual acuity; ERG: Electroretinogram; FA: Fluorescein angiography; ICGA: Indocyanine green angiography; OCTA: Optical coherence tomography angiography; PPRCA: Pigmented paravenous retinochoroidal atrophy; SD-OCT: Spectral domain optical coherence tomography
}

\section{Funding}

This study was supported by Project of National Key Research Program on Precision Medicine (2016YFC0904800) and Project of Science and Technology Committee of Shanghai (16140901003). No author has a financial or proprietary interest in any material or method mentioned.

Availability of data and materials

All date generated and analyzed during this study are included in this article.

\section{Authors' contributions}

$\mathrm{HC}$ and YS acquired data and drafted the article. XX drafted the article and analyze the data. YS obtained funding. All authors reviewed the manuscript. All authors read and approved the final manuscript.

Ethics approval and consent to participate No ethical approval required.

\section{Consent for publication}

Written informed consent was obtained from the patient for publication of this case report and any accompanying images. A copy of the written consent is available for review by the editor of this journal.

\section{Competing interests}

The authors declare that they have no competing interests.

\section{Publisher's Note}

Springer Nature remains neutral with regard to jurisdictional claims in published maps and institutional affiliations.

Received: 20 March 2018 Accepted: 30 May 2018

Published online: 07 June 2018

References

1. Choi JY, Sandberg MA, Berson EL. Natural course of ocular function in pigmented paravenous retinochoroidal atrophy. Am J Ophthalmol. 2006; 141(4):763-5.

2. Ahmed AS, Rishi P. A rare presentation of pigmented paravenous retinochoroidal atrophy. Oman J Ophthalmol. 2015;8(1):47-9.

3. Huang HB, Zhang YX. Pigmented paravenous retinochoroidal atrophy (Review). Exp Ther Med. 2014;7(6):1439-45.

4. Spaide RF, Klancnik JM Jr, Cooney MJ. Retinal vascular layers imaged by fluorescein angiography and optical coherence tomography angiography. JAMA Ophthalmol. 2015;133(1):45-50.

5. Mandadi SKR, Agarwal A, Aggarwal K, et al. Novel findings on optical coherence tomography angiography in patients with tubercular serpiginous-like choroiditis. Retina. 2017;37(9):1647-59.

Ready to submit your research? Choose BMC and benefit from:

- fast, convenient online submission

- thorough peer review by experienced researchers in your field

- rapid publication on acceptance

- support for research data, including large and complex data types

- gold Open Access which fosters wider collaboration and increased citations

- maximum visibility for your research: over $100 \mathrm{M}$ website views per year

At BMC, research is always in progress.

Learn more biomedcentral.com/submissions 